\title{
Topical Application of Exosomes Derived from Human Umbilical Cord Mesenchymal Stem Cells in Combination with Sponge Spicules for Treatment of Photoaging
}

This article was published in the following Dove Press journal:

International Journal of Nanomedicine

Keda Zhang, (iD ${ }^{1,2, *} \mathrm{Li}$ Yu, (iD) ${ }^{3, *}$ Fu-Rong $\mathrm{Li}$, (D) ${ }^{4} *$ Xue $\mathrm{Li}$, (D) ${ }^{5}$ Zhefeng Wang, (iD ${ }^{6}$ Xiaoting Zou, ${ }^{1,2}$ Chi Zhang, ${ }^{7}$ Kongpeng Lv, (iD) ${ }^{2}$ Boping Zhou,' Samir Mitragotri, ${ }^{8}$ Ming Chen (iD) ${ }^{7}$

'Central Laboratory of Longhua Branch, Second Clinical Medical College (Shenzhen People's Hospital) of Jinan University, Shenzhen 518109, People's Republic of China; ${ }^{2}$ Integrated Chinese and Western Medicine Postdoctoral Research Station, Jinan University, Guangzhou 51 0632, People's Republic of China;

${ }^{3}$ Department of Plastic and Aesthetic Surgery, Second Clinical Medical College (Shenzhen People's Hospital) of Jinan University, Shenzhen 518020, People's Republic of China;

${ }^{4}$ Translational Medicine Collaborative Innovation Center, Second Clinical Medical College (Shenzhen People's Hospital) of Jinan University, Shenzhen, 518020, People's Republic of China; ${ }^{5}$ Institute of Mass Spectrometry and Atmospheric Environment, Jinan University, Guangzhou 510632, People's Republic of China; ${ }^{6}$ Department of Cardiology, Second Clinical Medical College (Shenzhen People's Hospital) of Jinan University, Shenzhen 518020, People's Republic of China; ${ }^{7}$ StateProvince Joint Engineering Laboratory of Marine Bioproducts and Technology, College of Ocean and Earth Sciences, Xiamen University, Xiamen 361102, People's Republic of China; ${ }^{8}$ School of Engineering and Applied Sciences, Harvard University, Cambridge, MA 02138 USA

*These authors contributed equally to this work
Purpose: The topical application of exosomes secreted by mesenchymal stem cells (MSCExos) on the skin is a very new and interesting topic in the medical field. In this study, we aimed to investigate whether marine sponge Haliclona sp. spicules (SHSs) could effectively enhance the skin delivery of human umbilical cord-derived MSC-Exos (hucMSC-Exos), and further evaluate the topical application of hucMSC-Exos combined with SHSs in rejuvenating photoaged mouse skin.

Materials and Methods: SHSs were isolated from the explants of sponge Haliclona sp. with our proprietary method, and hucMSC-Exos were prepared from the conditioned medium of hucMSCs using ultracentrifugation. The effects of SHSs on the skin penetration of fluorescently labeled hucMSC-Exos were determined using confocal microscopy in vitro (porcine skin) and in vivo (mouse skin). The therapeutic effects of hucMSC-Exos coupled with SHSs against UV-induced photoaging in mice were assessed by using microwrinkles analysis, pathohistological examination and real-time RT-PCR. We also tested the skin irritation caused by the combination of hucMSC-Exos and SHSs in guinea pigs.

Results: In vitro results showed that hucMSC-Exos could not readily penetrate through porcine skin by themselves. However, SHSs increased the skin absorption of exosomes by a factor of 5.87 through creating microchannels. Similar penetration enhancement of hucMSCExos was observed after SHSs treatment in mice. The combined use of hucMSC-Exos and SHSs showed significant anti-photoaging effects in mice, including reducing microwrinkles, alleviating histopathological changes, and promoting the expression of extracellular matrix constituents, whereas hucMSC-Exos alone produced considerably weaker effects. Skin irritation test showed that the combination of hucMSC-Exos and SHSs caused slight irritation, and the skin recovered shortly.

Conclusion: SHSs provide a safe and effective way to enhance the skin delivery of MSCExos. Moreover, the combination of MSC-Exos and SHSs may be of much use in the treatment of photoaging.

Keywords: exosomes, mesenchymal stem cell, skin delivery, marine sponge spicules, photoaging

\section{Introduction}

Stem cells can renew themselves by cell division and differentiate into a variety of specialized cell types. Currently, a rapidly growing number of studies are being done to develop stem cell-based therapeutic strategies for many disorders and injuries within
Correspondence: Ming Chen; Boping

Zhou

Email ming.chen@xmu.edu.cn;

bopingzhou@163.com 
almost all parts of the body. ${ }^{1,2}$ Recent preclinical and clinical studies have demonstrated the efficacy of tissue-derived mesenchymal stem cells (MSC) transplantation in tissue repair and regeneration. ${ }^{2}$ Paracrine regulations of transplanted stem cells, in addition to the replacement of injured tissue cells by differentiation, are now known to contribute to the therapeutic effects. ${ }^{3,4}$

Exosomes are small lipid membrane extracellular vesicles ranging from 30 to $150 \mathrm{~nm}$ in diameter. ${ }^{5}$ These vesicles are secreted by nearly all eukaryotic cells through fusion of cytoplasmic multivesicular bodies (late endosomes) with the plasma membrane. ${ }^{6}$ They contain abundant nucleic acids (eg, mRNA and miRNAs), proteins (eg, various cytokines and growth factors) and lipids from parent cells, allowing them to participate in intercellular molecular exchange and communication. ${ }^{7}$ It has been widely reported that MSC-derived exosomes (MSCExos) can promote wound healing and tissue regeneration via enhancing fibroblast migration and proliferation and promoting angiogenesis as well as inducing endogenous stem cell recruitment. ${ }^{8}$ MSC-Exos are believed to be a promising cell-free therapy in regenerative medicine, with avoidance of tumorigenicity and immune rejection. ${ }^{9}$

The stratum corneum (SC), as the primary skin defensive layer against the external environment, is poorly permeable to hydrophilic compounds and biomacromolecules. ${ }^{10}$ It may be expected that functional constituents of exosomes, such as nucleic acids and proteins, cannot readily penetrate through the skin. Recently, Kim et al reported the topical application of human umbilical cord blood MSC-Exos on ex vivo human skin, which resulted in an increased expression of skin extracellular matrix (ECM) genes and thus benefited skin rejuvenation. ${ }^{11}$ We noted, however, that the authors did not examine the integrity of skin samples prior to use, especially in the case that skin samples were transported over a long distance. On the other hand, the absorption and efficacy of exosomes can be further improved by increasing skin permeability. Therefore, in order to develop the topical application of MSC-Exos, their skin absorption and potential penetration enhancing methods need to be further studied.

We have recently developed marine sponge Haliclona sp. spicules (SHSs) as a novel kind of microneedles for enhancing skin delivery of hydrophilic biomacromolecules. ${ }^{12}$ SHSs are silicious oxeas, which can penetrate the skin by simple massage and create over 1000 microchannels per $\mathrm{mm}^{2}$ in the skin. ${ }^{12}$ Also, it has been shown that the combination of SHSs and flexible liposomes may produce a synergic effect to dramatically improve the skin delivery of hyaluronic acid $(\sim 250 \mathrm{kDa}){ }^{13}$ Thus, we speculated that SHSs could facilitate the skin delivery of MSC-Exos and subsequently achieve the desired therapeutic results. In this study, we aimed to examine the skin absorption of exosomes derived from human umbilical cord MSCs (hucMSC-Exos), and more importantly to investigate whether SHSs could effectively deliver exosomes into the deeper skin layers. We further evaluated the topical application of hucMSCExos combined with SHSs in rejuvenating photoaged mouse skin. This study provided a useful strategy for realizing the therapeutic function of MSC-Exos through skin delivery, thus moving forward their translation from bench to bedside.

\section{Materials and Methods}

\section{Preparation and Characterization of SHSs}

SHSs were isolated and purified from the explants of marine sponge Haliclona sp., which was cultivated and collected at Dongshan Bay (Zhangzhou, China), according to our proprietary method (ZL201610267764.6). The surface topography of SHSs was visualized by using a scanning electron microscope (SEM; SIGMA, Carl Zeiss, Germany). The size distribution of SHSs was analyzed by measuring a random sample of 100 spicules by using a light microscope (BX51, Olympus, Japan) equipped with a digital camera (Olympus DP70) supported by ImageJ software v1.51a (NIH, USA).

\section{Cell Culture}

Human umbilical cord MSCs (hucMSCs) were purchased from Cyagen (Suzhou, China), and human dermal fibroblasts (HDFs) from Jennio (Guangzhou, China). HucMSCs were cultured in human MSC growth medium (Cyagen) and passaged when the confluency reaches approximately $80-90 \%$. At passage 6 , the culture medium was replaced with complete MesenCult ${ }^{\mathrm{TM}}-\mathrm{ACF}$ Plus Medium (STEMCELL Technologies, Hong Kong, China), which is a serum- and animal component-free culture medium, and collected for the isolation of exosomes at $90 \%$ cell confluency. HDFs were cultured and expanded in Dulbecco's modified Eagle medium (DMEM) containing $10 \%$ fetal bovine serum, penicillin $(100 \mathrm{U} / \mathrm{mL})$ and streptomycin $(100 \mathrm{mg} / \mathrm{mL})$, and used for the experiments over passages 5-9. All these cells were cultured in a humidified incubator under a $5 \% \mathrm{CO}_{2}$ atmosphere at $37^{\circ} \mathrm{C}$. 


\section{Isolation and Characterization of hucMSC-Exos}

HucMSC-Exos were isolated from the conditioned medium of hucMSCs using the classical ultracentrifugation method. ${ }^{14}$ The detailed procedures were shown in the Supplementary section. The size distribution and concentration of hucMSC-Exos were determined by nanoparticle tracking analysis (NTA) using ZetaView PMX 110 (Particle Metrix, Germany). Their protein content was measured by using the Pierce BCA protein assay kit (Thermo Fisher Scientific, USA), and protein markers (CD9, CD63, CD81 and calnexin) were determined by Western blot analysis. HucMSC-Exos was visualized by using a transmission electron microscope (TEM; Tecnai G2 Spirit BioTWIN, FEI, USA) and also a cryogenic transmission electron microscope (cryo-TEM; Titan Krios G3, FEI, USA).

\section{Internalization of hucMSC-Exos by HDFs}

HucMSC-Exos were labeled with DiR (1,1'-dioctadecyl3,3,3',3'-tetramethylindotricarbocyanine iodide; Invitrogen, Carlsbad, CA, USA), a near-infrared fluorescent membrane dye, and then precipitated using ExoQuick-TC ${ }^{\mathrm{TM}}$ (System Biosciences, USA). After centrifugation at $10,000 \times \mathrm{g}$ for 10 min, labeled exosomes were resuspended in PBS and stored at $-4{ }^{\circ} \mathrm{C}$. HDFs were seeded at $5 \times 10^{3}$ cells per well in 96 well glass bottom plates (CellVis, USA), and incubated with labeled exosomes $(250 \mu \mathrm{g} / \mathrm{mL})$ at $37^{\circ} \mathrm{C}$ for $4 \mathrm{~h}$. Control cells were treated with the culture medium. The cells were washed twice with PBS and then fixed with 4\% paraformaldehyde in PBS. The nuclei were counterstained with DAPI (4', 6-diamidino-2-phenylindole; Beyotime, Shanghai, China). Finally, the cells were photographed using a confocal microscope (TCS SP8, Leica Microsystems, Germany).

\section{Cell Proliferation Assay}

HDFs were plated into 96-well plates $\left(5 \times 10^{3}\right.$ per well) and incubated in growth medium for $24 \mathrm{~h}$. After 16-h starvation with serum-free DMEM, HDFs were washed with PBS and irradiated with UVB (280-360 nm, peak at $313 \mathrm{~nm}$ ) generated by a UV lamp (UVB-313EL, Q-Lab, USA) at $70 \mathrm{~mJ} / \mathrm{cm}^{2}$ (duration: around $6 \mathrm{~min}$ ). The irradiation dose was controlled by a digital UV meter (UV-340A, Lutron, Taiwan) whose detection wavelength ranges from $290 \mathrm{~nm}$ to $390 \mathrm{~nm}$. Then, the cells were cultured with hucMSC-Exos at different concentrations $(0,10$ and 30 $\mu \mathrm{g} / \mathrm{mL}$ ) in serum-free DMEM for $48 \mathrm{~h}$. The control group was not exposed to UV irradiation and treated with serumfree DMEM. Afterwards, CCK-8 solution (10 $\mu \mathrm{L}$; TransGen, China) was added into each well and incubated for $4 \mathrm{~h}$. The absorbance of each well was measured at $450 \mathrm{~nm}$ using a Spark 10M microplate reader (Tecan, Switzerland).

\section{Cellular Senescence Assay}

HDFs $\left(5 \times 10^{4}\right)$ were seeded per well in 6-well plates and cultured for $24 \mathrm{~h}$. The following procedures were conducted as same as cell proliferation assay. But after treatment with exosomes, the cells were fixed and stained using a senescence-associated $\beta$-galactosidase (SA- $\beta$-gal) staining kit (Cell Signaling Technology, USA) according to the product instruction. Four images were captured in each well in four directions (up, down, left and right) using an inverted light microscope (Eclipse Ts2, Nikon, Japan), and senescent cells were counted by blue staining.

\section{In vitro Skin Penetration Study}

Full-thickness porcine skin was obtained from Yinxiang Group Co., Ltd. (Xiamen, China). After removal of subcutaneous fat and hair shafts, the skin was cleaned with PBS and frozen at $-20^{\circ} \mathrm{C}$. Before experiments, skin disks (ø $28 \mathrm{~mm}$ ) were punched out from the frozen skin, and allowed to thaw at room temperature. The structural integrity of skin disks was checked by an electrical resistance test as described previously. ${ }^{15}$

Unjacketed Franz diffusion cells having an effective diffusion area of $1.54 \mathrm{~cm}^{2}$ and a receptor chamber volume of 15 $\mathrm{mL}$ were used to perform in vitro skin penetration experiments. Porcine skin disks were placed onto Franz cells with the SC upwards. The receptor chambers were fully filled with PBS and then maintained in a $37^{\circ} \mathrm{C}$ water bath. After 60 -min equilibrium, the air bubbles in the receptor medium were eliminated from the sampling port. HucMSC-Exos were labeled with a green fluorescent protein dye (ExoGlowProtein $^{\mathrm{TM}}$, System Biosciences, USA) as per the manufacturer's protocol. The skin disks were divided into four groups in terms of treatments on the skin prior to application of labeled exosomes, including untreated, massage, dermaroller and SHS groups. In the SHS group, the suspension of SHSs in PBS $(10 \mathrm{mg} / 100 \mu \mathrm{L})$ was applied onto the skin surface, followed by 2-min massage by an electrical massager (see Figure S1A) with a pressure force of about $0.3 \mathrm{~N}$ and a frequency of $300 \mathrm{r} / \mathrm{min}$. Meanwhile in the massage group, only 2-min massage was done on the skin. As for the dermaroller group, a dermaroller $(0.2 \mathrm{~mm}, 162$ microneedles; 
HC902, Dermaroller GmbH, Germany, see Figure S1B) was rolled over the skin in varying directions for $2 \mathrm{~min}$. No treatment was performed in the untreated group. Then, 150 $\mu \mathrm{L}$ of labeled hucMSC-Exos $(1 \mathrm{mg} / \mathrm{mL})$ was applied on the skin non-occlusively. After $16 \mathrm{~h}$, the residual exosomes on the skin surface were washed away with PBS six times. Small pieces of the skin were punched out from the skin disks and embedded in optimal cutting temperature (OCT) compound (Tissue-Tek ${ }^{\circledR}$, Sakura Finetek, USA) at $-80^{\circ} \mathrm{C}$. In addition, skin disks without treatments (including exosomes) were used as the blank to determine the autofluorescence of skin. Skin sections of $6-\mu \mathrm{m}$ thickness were cut by using a cryomicrotome (CM1860, Leica, Germany), and mounted on glass microscope slides in polyvinyl alcohol mounting medium with $\mathrm{DABCO}^{\circledR}$, antifading (Sigma-Aldrich, USA). The sections were photographed using a Leica TCS SP8 confocal microscope. Fluorescence intensity of each layer in the skin was assessed by using ImageJ software.

\section{Animals}

Female specific pathogen-free (SPF) Kunming mice (4-5 weeks old) were obtained from Guangdong Medical Laboratory Animal Center (Foshan, China), and female SPF guinea pigs (12 weeks old) were from Shanghai SLAC laboratory Animal Co., Ltd. (Shanghai, China). They were allowed to acclimatize for one week in the laboratory. All protocols were approved by the Animal Ethics Committee of Shenzhen People's Hospital (LLKY-2019067) and conducted in full compliance with the Guide of the Care and Use of Laboratory Animals of China.

\section{Photoaged Mouse Model and Exosome Treatments}

Photoaged mouse models were established by ten-week UV irradiation provided by the combination of one UVA lamp (315-400 nm, with a peak emission at $340 \mathrm{~nm}$ ) and two UVB lamps (280-360 nm, peak at $313 \mathrm{~nm}$ ). The experimental procedures were detailed in the Supplementary section. The photoaged mice were randomly grouped ( $\mathrm{n}=6$ per group): Model, SHS-PBS, Exo (H), SHS-Exo (L) and SHS-Exo (H). Anti-leakage plastic rings (inner diameter: $15 \mathrm{~mm}$, see Figure S1C) were attached to the backs of photoaged mice (except the Model group) using Vetbond ${ }^{\mathrm{TM}}$ tissue adhesive (3M, USA, see Figure S1D) in order to conveniently administer liquid formulations. In the SHS-Exo $(\mathrm{H})$ group, $150 \mu \mathrm{L}$ of
hucMSC-Exos $(1 \mathrm{mg} / \mathrm{mL})$ was evenly dripped into the plastic ring, which was then sealed by Tegaderm ${ }^{\mathrm{TM}}$ transparent film dressing (3M, USA, see Figure S1E), after SHSs $(10 \mathrm{mg})$ suspended in PBS $(100 \mu \mathrm{L})$ pierced the skin through 2-min electrical massage. The procedure was repeated every other day for two weeks. As to the SHSExo (L) and SHS-PBS groups, hucMSC-Exos $(150 \mu \mathrm{L}$, $100 \mu \mathrm{g} / \mathrm{mL})$ and PBS $(150 \mu \mathrm{L})$ were used instead, respectively. The Exo $(\mathrm{H})$ group was treated with hucMSC-Exos $(150 \mu \mathrm{L}, 1 \mathrm{mg} / \mathrm{mL})$ without SHSs, and no treatment was implemented in the Model group. In the meanwhile, one group of six normal mice was used as the control (Normal). One week after the final administration of exosomes, the surface of the dorsal skin enclosed by the plastic ring was imaged using an optical skin detection system at 50× magnification (WAX-PF012, Wesipull ${ }^{\mathrm{TM}}$, Hoppen, China). Then, the mice were sacrificed using $\mathrm{CO}_{2}$ gas and the dorsal skin specimens were harvested. Small skin pieces were taken from these specimens for histological examination (Supplementary section), and the rest of them were used for quantification of aging-related gene expressions using real-time reverse transcription-polymerase chain reaction (RT-PCR) (Supplementary section). The sequences of primers for real-time RT-PCR are listed in Table S1.

\section{Skin Irritation Study}

Anti-leakage plastic rings of $15 \mathrm{~mm}$ in inner diameter were one-to-one stuck to the shaved backs of guinea pigs using Vetbond ${ }^{\mathrm{TM}}$ tissue adhesive. The suspension of SHSs in PBS $(10 \mathrm{mg} / 100 \mu \mathrm{L})$ was applied to the skin area surrounded by the plastic ring, coupled with 2-min electrical massage. HucMSC-Exos $(150 \mu \mathrm{L}, 1 \mathrm{mg} / \mathrm{mL})$ were pipetted into the plastic ring, which was then sealed by transparent film dressing. The Draize assessment method was adopted to score the extents of erythema and edema over $72 \mathrm{~h}$ after the combined use of hucMSC-Exos and SHSs, ${ }^{16}$ and primary irritation index (PII) was calculated according to the method developed by Liu et al. ${ }^{17}$ The animals were sacrificed, and the skin tissues both untreated and treated were harvested from each guinea pig for histopathological analysis (see Supplementary section).

\section{Statistical Analysis}

All data were presented as mean \pm standard deviation (SD). Statistical analysis was performed using one-way analysis of variance, followed by a Student's $t$-test. A $p$ value $<0.05$ indicates significant difference. 


\section{Results}

\section{Characterization of SHSs and hucMSC- Exos}

SHSs of high purity (batch 19) were extracted from the explants of marine sponge Haliclona sp. cultivated and collected in 2019 (Figure 1A). The SHSs were smooth oxeas which had a rod-like body with sharp ends (Figure 1B). They had a cylinder diameter of $7.26 \pm 1.15$ $\mu \mathrm{m}$ and a length of $126.35 \pm 5.27 \mu \mathrm{m}$, which is almost same as those of batches 17 and 18 (cultivated in 2017,
2018), see Figure 1C. Batch 19 was used in this whole study. HucMSC-Exos were isolated from the conditioned medium of hucMSCs using ultracentrifugation. ${ }^{14}$ The morphology of HucMSC-Exos was characterized by both TEM with negative staining and cryo-TEM. TEM images showed a cup-shaped appearance (Figure 2A and B), while cryo-TEM revealed a round/spherical vesicular structure (Figure 2C). The contradictory observations were in good agreement with the previous reports. ${ }^{18}$ The cup shape has been considered as an artefact generated by the sample

\section{A}

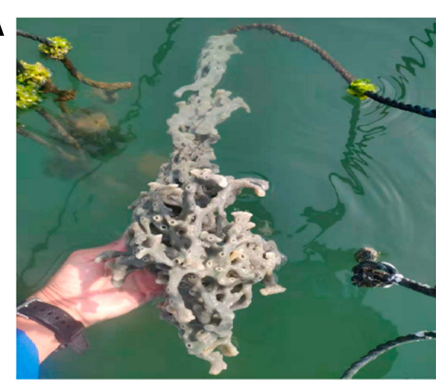

B

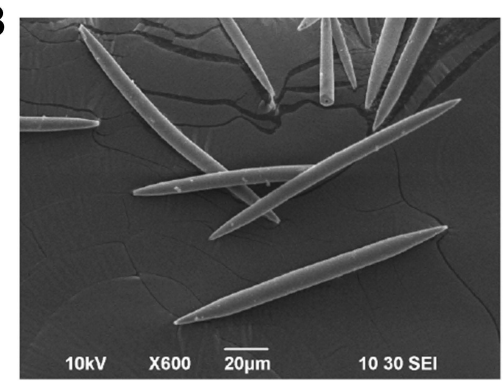

C

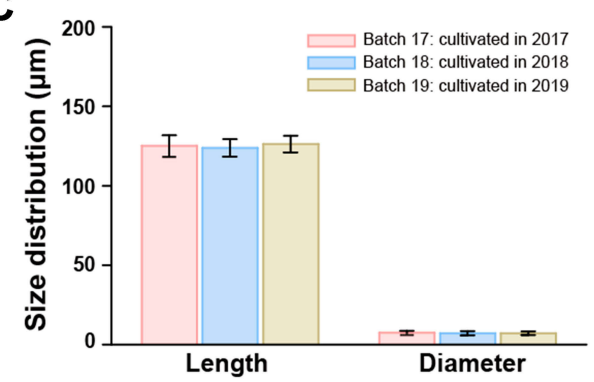

Figure I Origin and characterization of SHSs. (A) Explants of marine sponge Haliclona sp. cultivated in Dongshan Bay, China. (B) SEM photograph of SHSs. (C) The size distribution of SHSs isolated from three different batches.
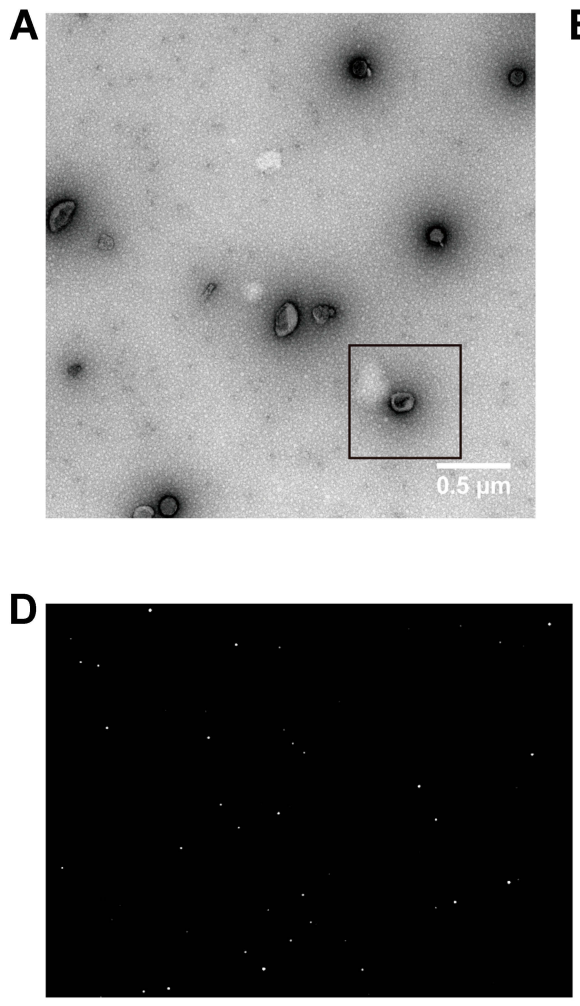

B
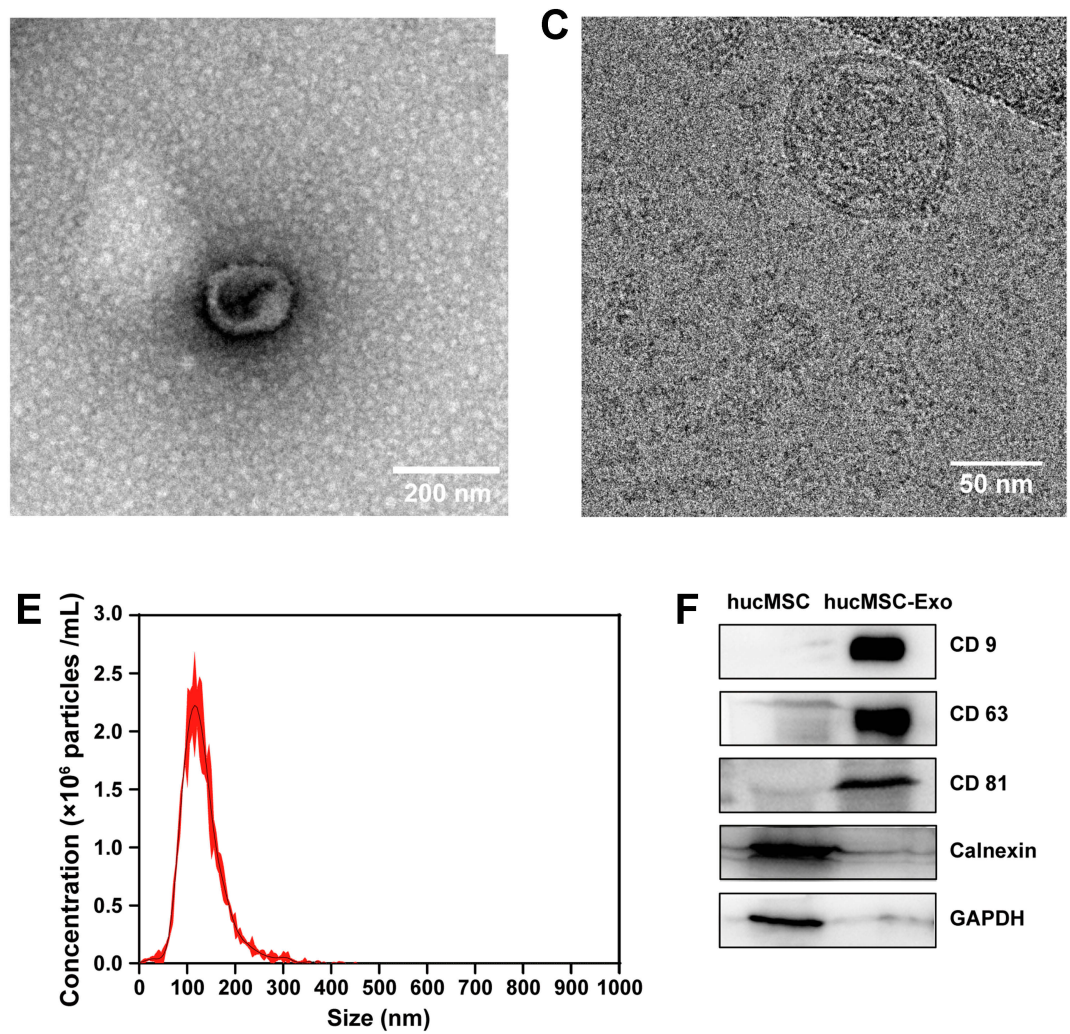

F

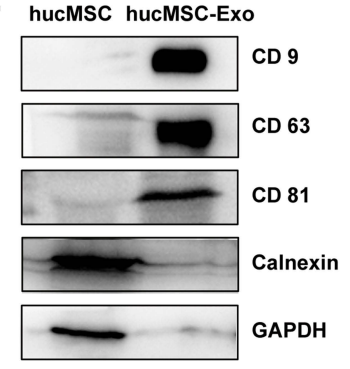

Figure 2 Characterization of hucMSC-Exos. (A) TEM photograph of hucMSC-Exos with negative staining using $2 \%$ uranyl acetate. (B) Enlarged view of the selected area (black box) in the image (A). (C) Cryo-TEM photograph of hucMSC-Exos. (D) NTA video image of hucMSC-Exos' movement under Brownian motion. (E) Size distribution measurement of hucMSC-Exos by NTA analysis. (F) Western blot analysis of protein markers of hucMSC-Exos. 
preparation steps, such as fixation and/or contrasting, and the innate shape of exosomes is spherical. The size distribution and concentration of HucMSC-Exos were measured by using NTA (Figure 2D). The exosomes ranged from $57.5 \mathrm{~nm}$ to $317.5 \mathrm{~nm}$ in diameter with a peak at 121.7 $\mathrm{nm}$ (Figure 2E). The concentration of exosomes was 1.53 $\times 10^{11}$ particles $/ \mathrm{mL}$. Western blot analysis confirmed the presence of exosomal protein markers, such as CD9, CD63, and CD81, and the absence of a negative control, calnexin (Figure 2F). Thus, the isolated vesicles were indeed exosomes.

\section{Suppressive Effects of hucMSC-Exos Against UV-Induced Damage in HDFs}

To examine the internalization of hucMSC-Exos, HDFs were incubated with membrane dye DiR-labeled hucMSC-Exos at $37^{\circ} \mathrm{C}$ for $4 \mathrm{~h}$ (see Supplementary section). Obvious red fluorescence was detected in the perinuclear region of HDFs, while the control treated with PBS had no fluorescence (Figure 3A). This suggested that hucMSC-Exos can be internalized by HDFs. The effects of hucMSC-Exos on the proliferation of UV-irradiated HDFs were investigated using CCK-8 assay. UV irradiation $\left(70 \mathrm{~mJ} / \mathrm{cm}^{2}\right)$ significantly reduced the proliferation of HDFs, but a 48-h treatment with hucMSC-Exos restored their proliferation in a dosedependent manner (Figure 3B). SA- $\beta$-gal is a widely used biomarker for senescent and aging cells. Senescent HDFs were stained blue through the cleavage of the chromogenic substrate X-Gal (5-bromo-4-chloro-3-indolyl- $\beta$-D-galactopyranoside) catalyzed by SA- $\beta$-gal. The proportion of senescent cells (89.2\%) was significantly higher in the UV-exposed group compared to the unexposed group (62.3\%), and the former was decreased effectively by hucMSC-Exos in a dose-dependent manner (Figure 3C and D). The number of cells in each microscopic image was counted manually (Figure 3C and E), displaying a similar variation trend to the CCK-8 assay among the groups. We also checked the effects of the exosomes on the expression of dermal ECM-associated genes in UV-irradiated HDFs by using real-time RT-PCR (Supplementary section). The mRNA levels of collagen I, elastin and fibronectin were down-regulated and that of matrix metalloproteinase-1 (MMP-1) was up-regulated in HDFs after UV exposure. Such a change was markedly suppressed or even reversed by hucMSC-Exo treatment (Figure 3F). All these results demonstrate that hucMSC-Exos have suppressive effects against UV-induced damage in HDFs.

\section{Enhanced Skin Delivery of hucMSC-Exos Coupled with SHSs}

We further investigated the enhancement effect of SHSs on the skin delivery of hucMSC-Exos in vitro. The skin penetration of exosomes was enhanced significantly by SHSs (Figure 4A), whereas dermaroller or massage appeared not to exert any obvious effect on their penetration. The untreated skin was used as the blank control to represent the skin autofluorescence. The accumulation of exosomes in each layer of the skin was semi-quantified by the fluorescent signal analysis using the image software, ImageJ (Figure 4B). The topical application of SHSs increased the accumulation of exosomes by a factor of 2.77 in the SC, 7.02 in the viable epidermis and 5.77 in the dermis, leading to an increase by a factor of 5.87 in the total skin absorption. The vast majority of exosomes delivered by SHSs were present in the deeper skin layers (viable epidermis and dermis). Besides, the skin absorption of exosomes through dermaroller and massage was 1.20 and 1.15 times, respectively, that in the untreated skin. These results indicated that SHSs are considerably more effective than the commercial dermaroller in enhancing the skin delivery of hucMSC-Exos. In addition, the skin delivery of exosomes based on SHSs was studied under non-occlusive condition in the anesthetized mice (Supplementary section). The skin absorption of exosomes was also enhanced significantly by SHSs in vivo (Figure 4C).

\section{Anti-Aging Effects of hucMSC-Exos Combined with SHSs in Photoaged Mice}

To investigate the anti-photoaging effects of hucMSCExos combined with SHSs in vivo, photoaged mouse model was established by UV irradiation for ten weeks. Obvious wrinkles were observed on the dorsal skin of irradiated mice compared to normal (or non-irradiated) mice (Figure 5A). Microwrinkles are wrinkles at the dimensions of skin microrelief, which contribute to the formation of visual wrinkles. ${ }^{19,20}$ A further microscopic photography showed the existence of many microwrinkles on irradiated skin and none of them on normal skin (Figure 5B). After different treatments for two weeks, the hucMSC-Exos combined with SHSs significantly improved the microwrinkles on the skin, while only SHSs or hucMSC-Exos hardly gave rise to conspicuous improvement (Figure 5B). Histopathological examination was performed on the skin tissues (Figure 5C). Model (or 
A

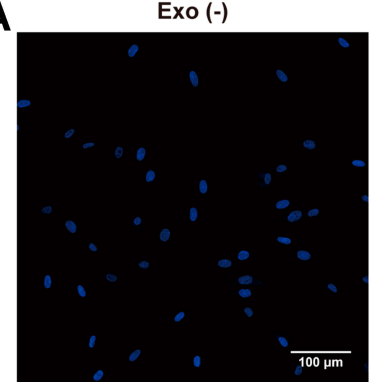

C

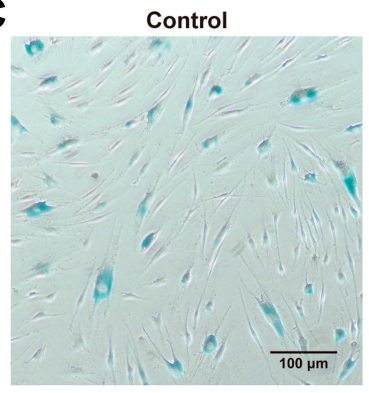

UV-Exo $(10 \mu \mathrm{g} / \mathrm{mL})$

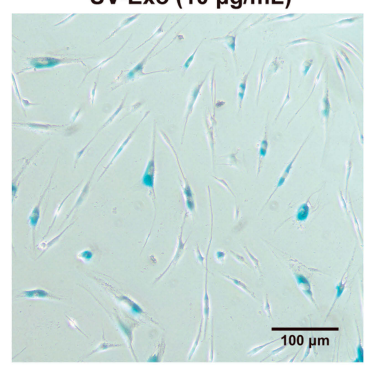

F
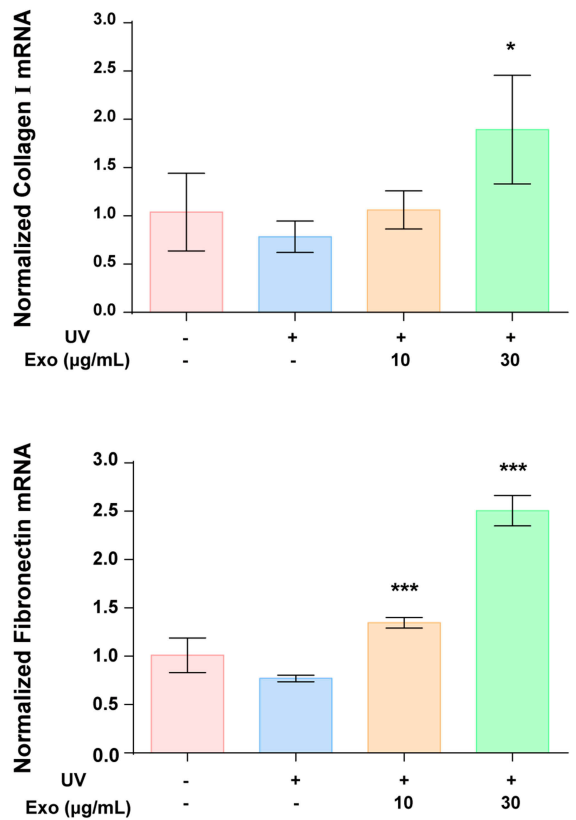

B

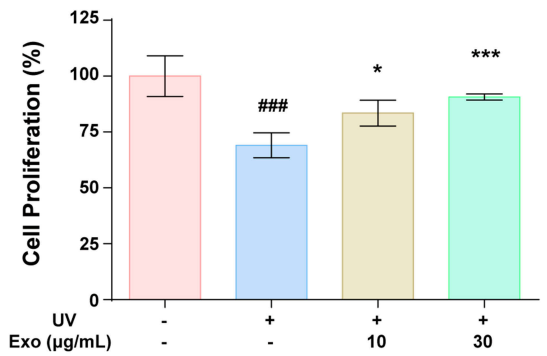

D

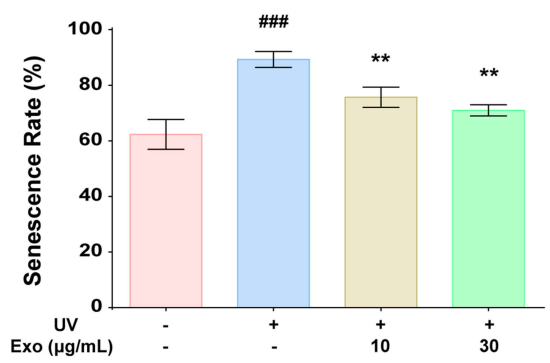

E
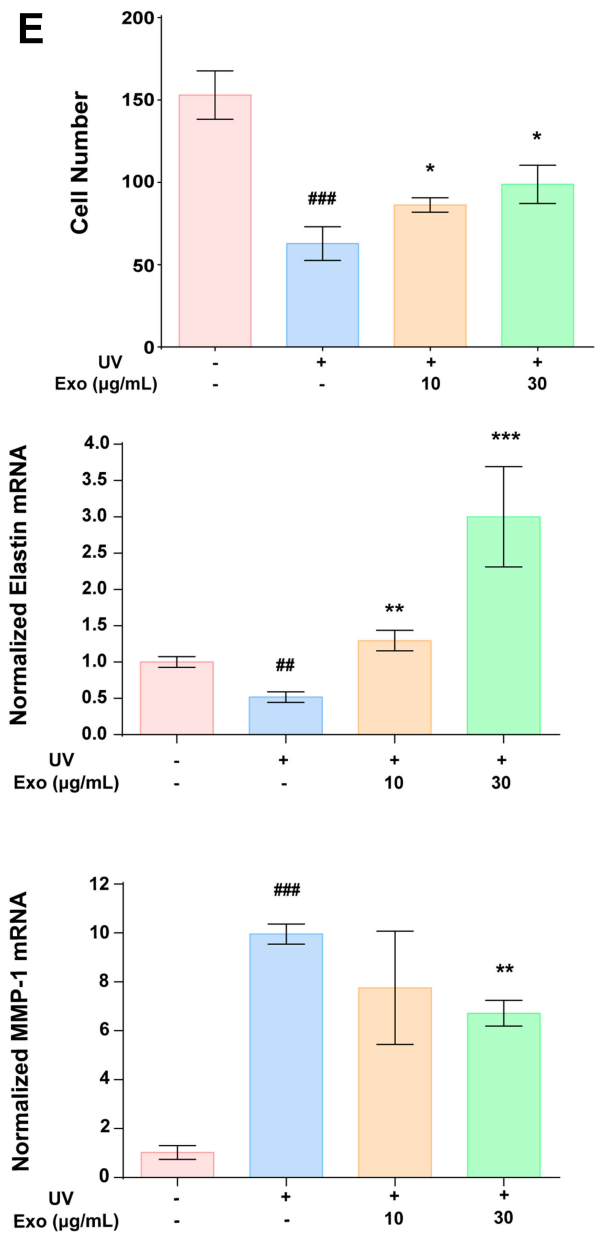

Figure 3 Effects of hucMSC-Exos on UV-induced damage in HDFs. (A) Confocal microscopic observation on the internalization of DiR-labeled hucMSC-Exos (red) by normal HDFs (stained with DAPI). (B) Effects of hucMSC-Exos on the proliferation of UV-irradiated HDFs (CCK-8 assay). (C) Effects of hucMSC-Exos on the expression of the biomarker for cellular senescence, SA- $\beta$-gal (blue staining). (D) Percentages of senescent cells in the photographs (C). (E) Numbers of cells in the photographs (C). (F) Effects of hucMSC-Exos on the mRNA expressions of dermal ECM-related genes in UV-irradiated HDFs. All data were expressed as mean $\pm S D(n=3)$. $* p<0.05$, $* * p<0.01$, *** $p<0.00$ I, versus the UV group; ${ }^{\#} p<0.01,{ }^{\# \#} p<0.001$, versus the control group. 

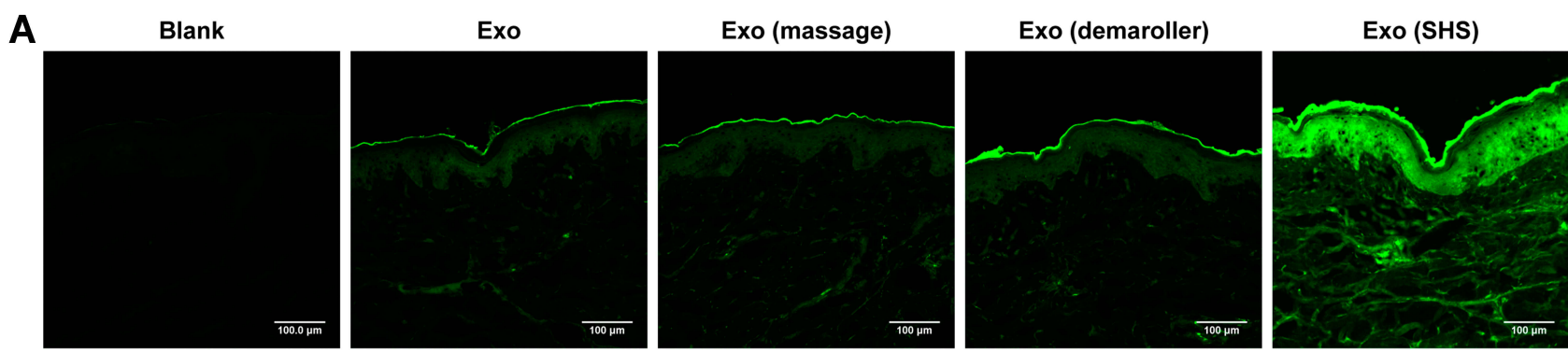

B

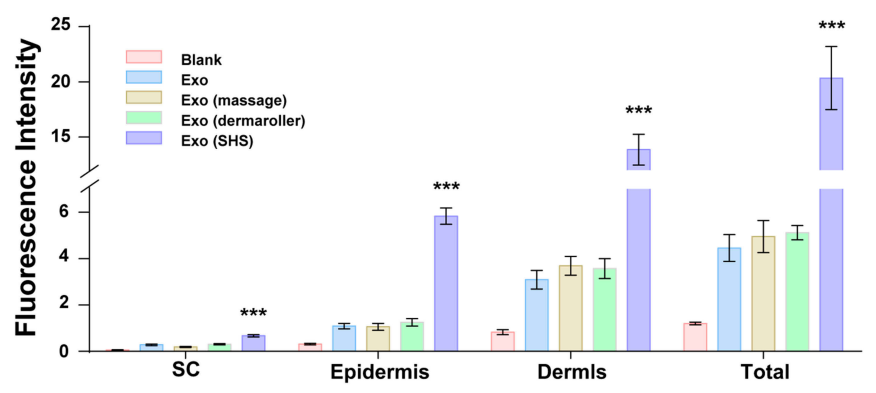

C
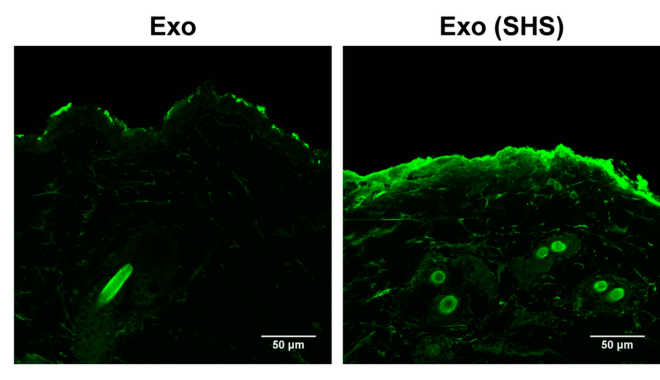

Figure 4 Effects of SHSs on the skin penetration of hucMSC-Exos in vitro and in vivo. (A) Penetration of fluorescent protein dye-labeled hucMSC-Exos (green) through porcine skin after 2-min massage by an electrical massager, 2-min application of a dermaroller, treatment of SHSs via 2-min massage by an electrical massager, and no treatment, respectively. The untreated skin was used as the blank to assess the autofluorescence of skin. (B) Deposition of dye-labeled hucMSC-Exos in each layer of skin. (C) Penetration of fluorescent protein dye-labeled hucMSC-Exos (green) through mouse skin treated with SHSs or untreated in vivo. All data were expressed as mean \pm SD $(\mathrm{n}=3) . * * * p<0.001$, versus the untreated exosome group.

photoaged) mouse skin was characterized by epidermal hyperplasia and flattening of the dermal-epidermal junction (DEJ), as reported previously. ${ }^{21,22}$ Epidermal hyperplasia, as one of the main reasons for wrinkle formation, ${ }^{23}$ was simply evaluated by measuring the epidermal thickness (Figure 5D; Supplementary section). The administration of hucMSC-Exos based on SHSs remarkably ameliorated the skin histopathological alterations induced by UV exposure. The SHS-Exo $(\mathrm{H})$ group even exhibited histopathological features very comparable to the normal group, which had thin layer of epidermis and wavy DEJ. Meanwhile, the SHS-PBS and Exo (H) groups had similar features to the model group.

In addition, the mRNA expressions of dermal ECMassociated genes were determined using real-time RT-PCR (Figure 5E). The results indicated a decrease in the mRNA levels of collagen I, elastin, and fibronectin and an increase in that of MMP-1 (collagenase) in the photoaged skin as compared to the normal skin. The combined use of hucMSC-Exos and SHSs produced a dose-dependent suppression on the abnormal gene expressions; hucMSC-Exos or SHSs alone apparently having much less influence. All these results suggested that hucMSC-Exos could not well penetrate through the skin, while the topical use of SHSs facilitated the skin delivery of exosomes and subsequently realized their anti-aging functions.

\section{Skin Irritation Test of the Combination of hucMSC-Exos and SHSs}

Guinea pigs were observed for signs of skin erythema and edema for $72 \mathrm{~h}$ after exposure to the combination of hucMSCExos $(150 \mu \mathrm{g})$ and SHSs (10 mg) (Figure 6). No perceptible irritation was seen in the guinea pig skin immediately following the treatment with hucMSC-Exos and SHSs. However, there was very slight erythema present at $24 \mathrm{~h}$, and then the symptom started to decrease until complete disappearance at $72 \mathrm{~h}$. The PII value (1.05) was within the grade of slight irritation (0.5-1.9), suggesting that the combined use of hucMSC-Exos and SHSs was slightly irritating. Furthermore, the treated skins of guinea pigs at different time points were harvested and histopathologically examined (Figure 7A). No evident morphological change of epidermal cells was observed in the skins. However, inflammatory infiltrate was observed in the upper dermis. The skin cells in a certain section area of dermis $(450 \mu \mathrm{m} \times 150 \mu \mathrm{m})$ were counted using ImageJ software. The number of skin cells reached the largest immediately after treatment, and then returned to and remained at the normal level after $24 \mathrm{~h}$ (Figure 7B).

\section{Discussion}

The valuable therapeutic potential of MSC-Exos in wound healing, tissue repair and regeneration make themselves gain 
A

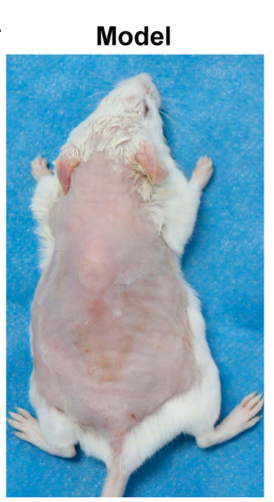

C

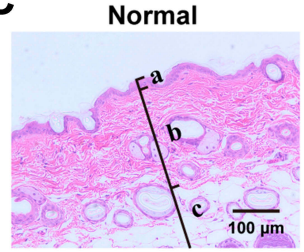

Exo (H)

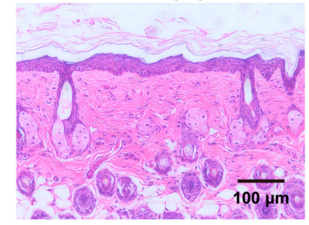

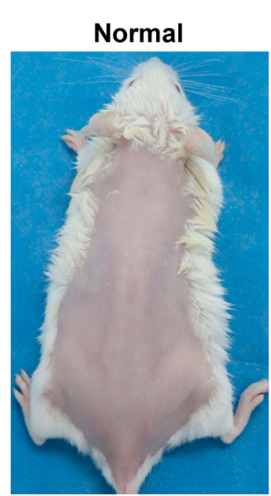

Model

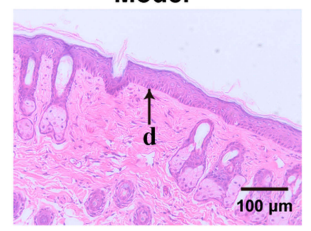

SHS-Exo (L)

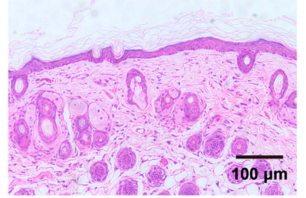

B

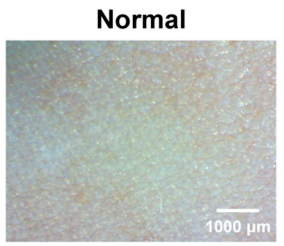

Exo (H)

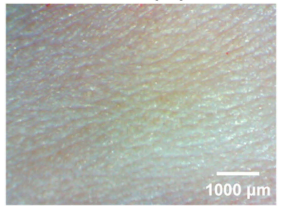

SHS-PBS

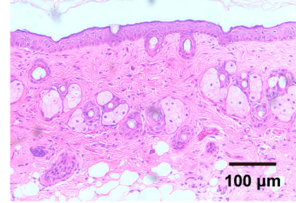

SHS-Exo (H)

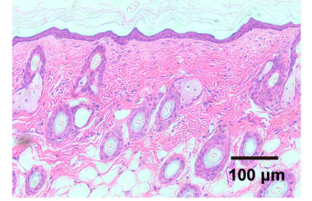

Model

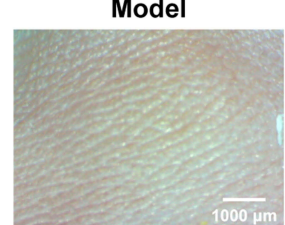

SHS-Exo (L)

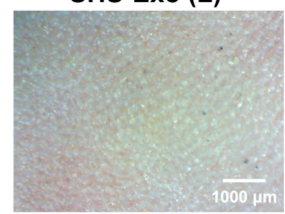

D

E
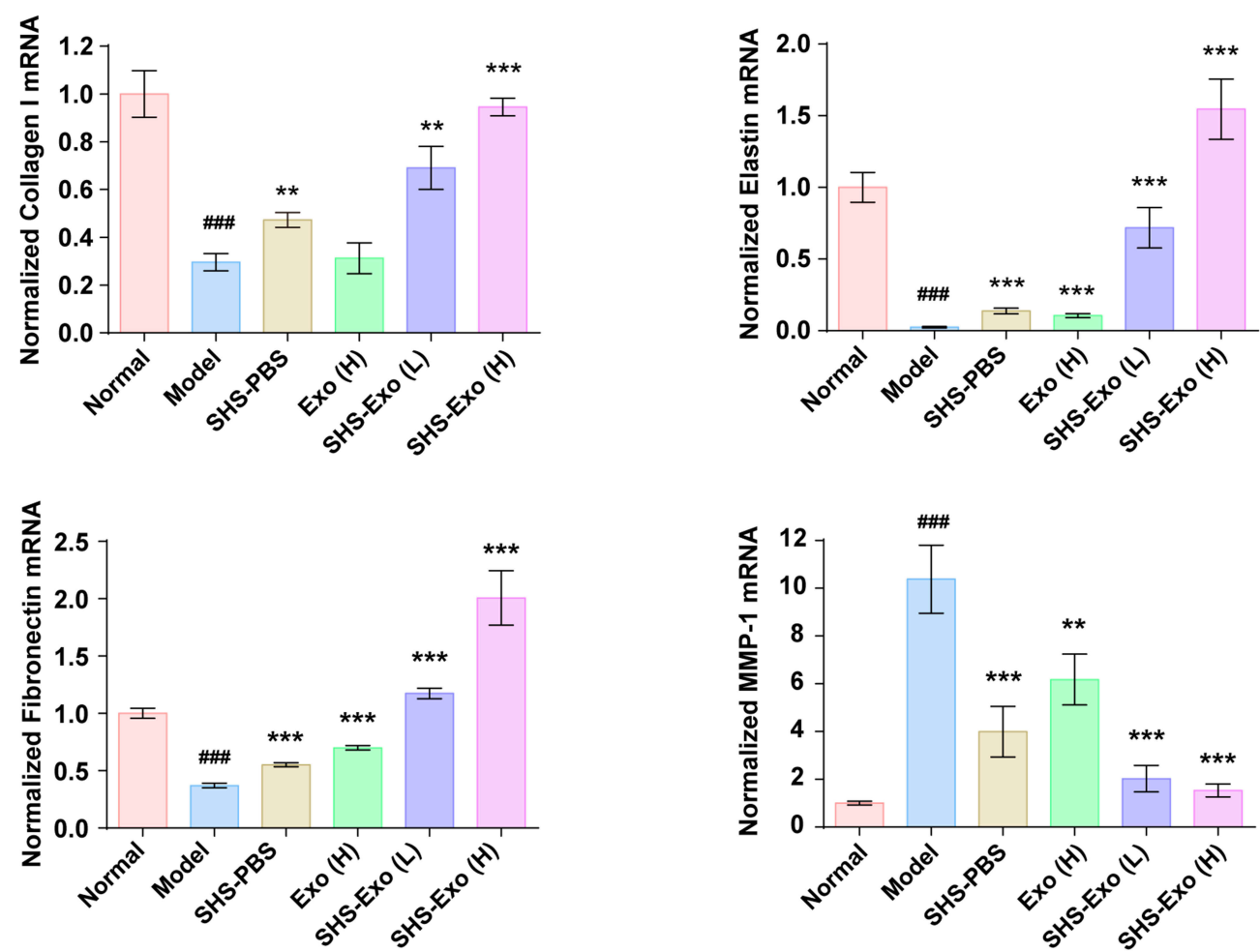

Figure 5 Enhanced anti-aging effects of hucMSC-Exos on photoaged mouse skin by using SHSs. (A) Macroscopic observation of photoaged skin induced by UV-irradiation in mice. (B) Changes in microwrinkles of photoaged skin by different treatments. (C) Histopathological analysis (H\&E staining) of photoaged skin after different treatments. Labels: a, epidermis; b, dermis; c, subcutaneous tissue; d, dermal-epidermal junction. (D) Epidermis thickness of photoaged skin after treatments. (E) mRNA expressions of dermal ECM-related genes after treatments. All data were expressed as mean \pm SD $(n=4)$. $*_{p}<0.05$, **p $<0.01$, ***p $<0.00$ I, versus the model group; ${ }^{*} p<0.00$ I, versus the normal group. 

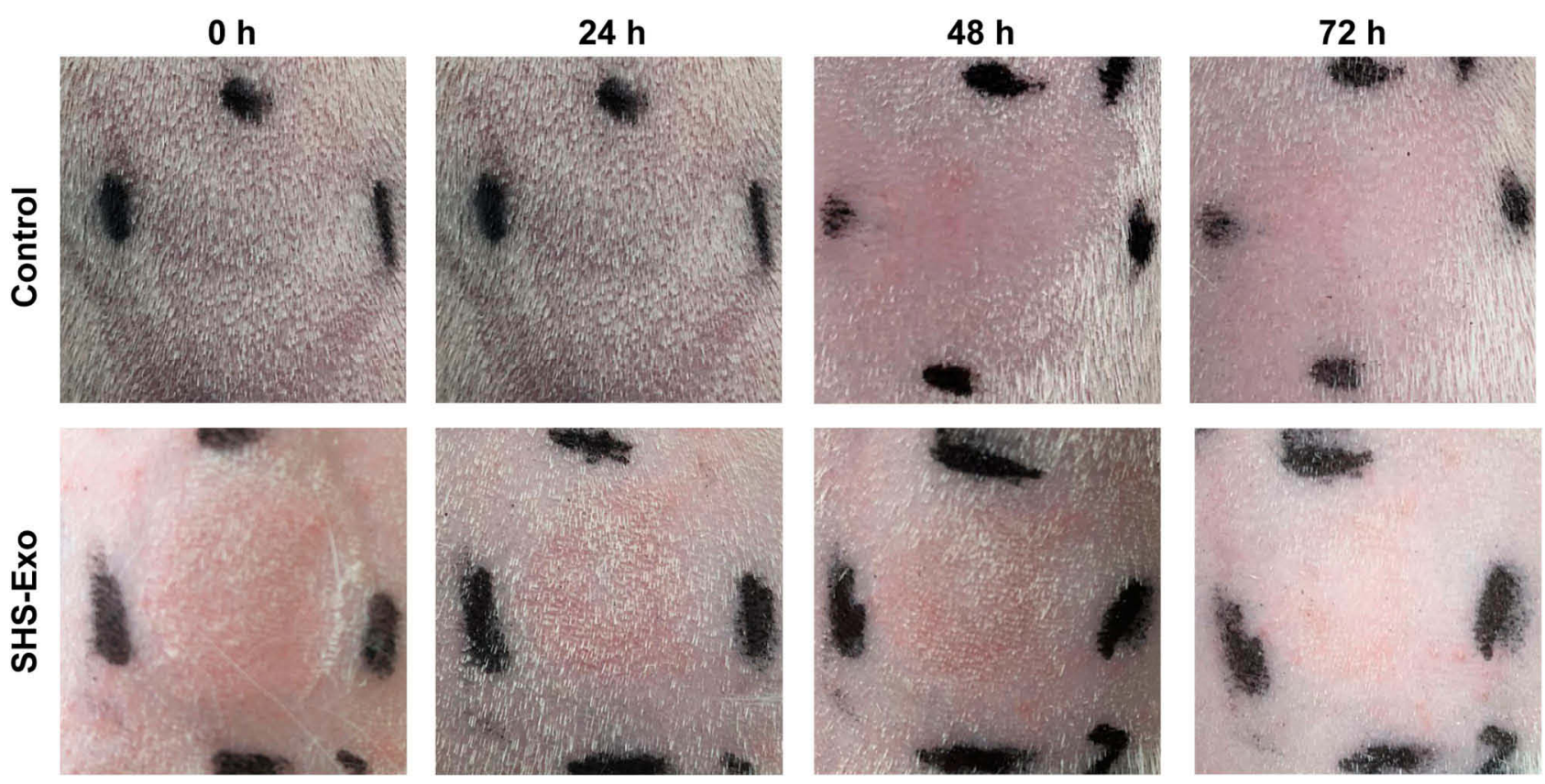

Figure 6 Skin irritation response of guinea pigs to the combination of hucMSC-Exos Exos ( $150 \mu \mathrm{L}, 1 \mathrm{mg} / \mathrm{mL})$ and SHSs over $72 \mathrm{~h}$.

A

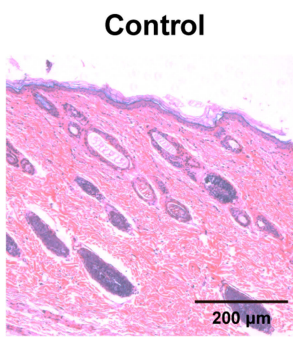

$48 \mathrm{~h}$

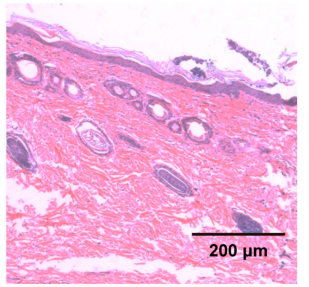

$\mathrm{Oh}$

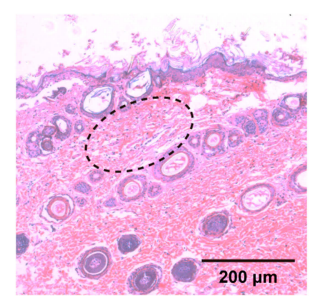

$72 \mathrm{~h}$

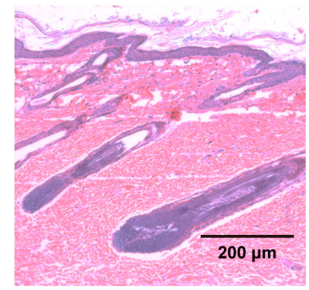

$24 \mathrm{~h}$

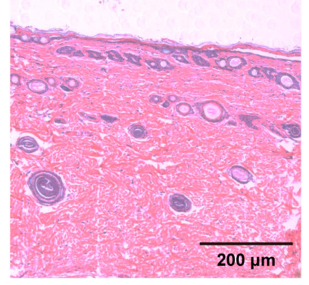

B

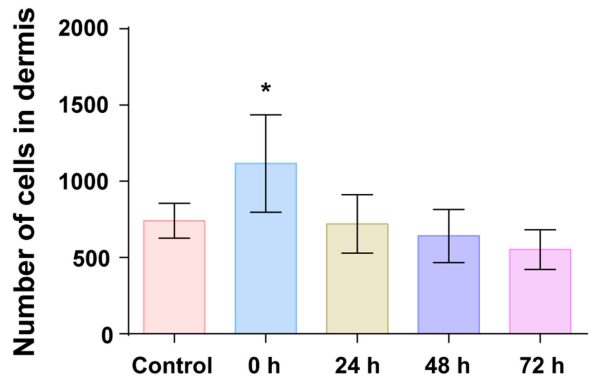

Figure 7 Histopathological analysis of guinea pig skins after the combined use of hucMSC-Exos (I50 $\mu \mathrm{L}, \mathrm{I} \mathrm{mg} / \mathrm{mL})$ and SHSs over $72 \mathrm{~h}$. (A) Histopathological images of the skins. Dashed circle: inflammatory infiltration. (B) Numbers of cells in a certain section area $(450 \mu \mathrm{m} \times 150 \mu \mathrm{m})$ of dermis. All data were expressed as mean $\pm \mathrm{SD}(\mathrm{n}=3)$. ${ }^{p}$ $<0.05$, versus the control group.

growing interest in translational medicine. However, owing to the existence of hydrophobic SC, the skin is poorly permeable to hydrophilic biomacromolecules (such as proteins and RNAs) bound or capsulated in exosomes. Further, it has been confirmed by lots of previous studies that conventional liposomes, made up of phospholipids with or without cholesterols, cannot penetrate through the SC as entities but fuse with the lipid matrix, leading to merely a deposition at the surface of the skin. ${ }^{24,25}$ In theory, exosomes, which have the same enclosed lipid bilayer structure as conventional liposomes, cannot penetrate into the deeper skin layers. This is evidenced by our observations that weak fluorescent signals were detected in the deeper layers of skin after the application of protein dye-labeled hucMSC-Exos both in vitro and in vivo (Figure 4). Moreover, the normal skin absorption of hucMSCExos failed to produce the desired anti-aging effects on 
photoaged skin in mice (Figure 5). Therefore, enhanced skin delivery is required for MSC-Exos topically applied to realize their therapeutic function.

Microneedles have been developed as a very promising technology for improving the skin delivery of biotherapeutics and even nanoparticles. ${ }^{26-28}$ A growing number of human clinical trials indicate good safety, efficacy and patient acceptance of microneedles. ${ }^{29,30}$ Recently, we reported that SHSs could be used as a novel microneedle system to promote the skin absorption of hydrophilic macromolecules. $^{12}$ SHSs can readily pierce the skin through gentle massage, and $10 \mathrm{mg}$ of them may create more than 1000 microchannels (with a depth of $42.2 \pm$ $14.9 \mu \mathrm{m}$ ) per $\mathrm{mm}^{2.12}$ SHSs (or the microchannels caused) will gradually disappear from the skin within three days due to normal desquamation. Very importantly, SHSs may be suited to any desired skin location and area. In contrast, the most studied microneedle patches can only be applied in a limited and flat skin region (often 1-10 $\mathrm{cm}^{2}$ ) according to the design of products; ${ }^{26}$ the first microneedle product, dermaroller (solid microneedle roller), only provides transient open microchannels, which gradually close up in $30 \mathrm{~min}$ after microneedle treatment due to the skin contraction. ${ }^{31}$ And these microneedle systems usually generate a much smaller number of microchannels $\left(10^{2}-10^{4}\right)$ per unit skin area $\left(\mathrm{cm}^{2}\right)$ as compared to SHSs. ${ }^{32}$ In view of the advantages of SHSs, we here investigated whether SHSs can enhance the skin delivery of MSC-Exos.

HucMSC-Exos were nearly six times more absorbed into the skin in the porcine skins treated with SHSs than in the untreated skins in vitro (Figure 4B). Moreover, SHSs significantly increased the accumulation of hucMSC-Exos in all skin layers, in particular in the deeper skin layers. Similar skin penetration behaviors of hucMSC-Exos were observed with or without SHS treatment in mice (Figure 4C). This suggests that exosomes can penetrate into the deeper layers of skin through the microchannels created by SHSs. On the other hand, the commercial dermaroller hardly improved the skin absorption of exosomes, which may be explained by a short-period opening of the microchannels caused. These findings demonstrate that SHSs can be an effective approach to enhancing the skin delivery of MSC-Exos.

One inevitably undergoes intrinsic and extrinsic aging of the skin throughout the whole life. The former is determined by individual genetics, and the latter is caused by various external factors, among which UV-exposure is ranked at the top. ${ }^{33}$ Previously, Oh et al reported that human-induced pluripotent stem cells-derived exosomes ameliorated UVB-induced photoaging and natural senescence of HDFs. $^{34}$ In this study, we found that hucMSCExos were internalized by HDFs (Figure 3A), and rescued the cells from the damage induced by UV exposure. Specifically, hucMSC-Exos promoted the proliferation of UV-irradiated HDFs in a dose-dependent manner (Figure 3B). HucMSC-Exos significantly decreased the proportion of senescent cells in the UV-irradiated HDFs (Figure 3C and D). In addition, rebuilding of dermal ECM, which requires regeneration of ECM constituents, plays an essential role in rejuvenation of aged skin. ${ }^{35,36}$ The treatment with hucMSC-Exos reversed the downregulation of dermal ECM constituents (collagen I, elastin and fibronectin) and suppressed the upregulation of the fibroblast collagenase (MMP-1) caused by UV irradiation (Figure 3F). Collectively, hucMSC-Exos can effectively repair the UV damage in HDFs, and thus possess the therapeutic potential against skin photoaging.

MSC-Exos have been recognized as potent therapeutics in tissue repair and inflammation suppression as well as anticancer therapy. ${ }^{37}$ Current studies have suggested that MSC-Exos generate the therapeutic effects largely via the transfer of miRNAs and proteins, which trigger diverse signalling pathways in the recipient cells. ${ }^{38} \mathrm{Ti}$ et al reported 15 upregulated miRNAs present in hucMSCExos compared to human fibroblast-derived exosomes. Among them, three miRNAs (miR-21, miR-146a, and miR-181) exhibited the most significant difference and the highest expression in hucMSC-Exos and may contribute to resolution of wound inflammation and tissue repair. $^{39}$ Liang et al demonstrated that human adipose MSC-Exos could transfer miR-125a to endothelial cells and promote angiogenesis through suppressing the expression of angiogenic inhibitor delta-like 4 (DLL4). ${ }^{40}$ Also, Kim et al showed that human umbilical cord blood MSCExos containing high amount of growth factors (especially EGF and bFGF) associated with skin rejuvenation stimulated fibroblast proliferation and migration as well as collagen and elastin synthesis. ${ }^{11}$ Moreover, many evidences show that MSC-Exos benefit some key steps in wound repair and cutaneous regeneration, such as cell migration and proliferation, angiogenesis and collagen deposition, mainly by activation of AKT/ERK and Wnt signaling. ${ }^{41}$ So presumably, the beneficial effects of hucMSC-Exos on UV-damaged HDFs result from the synergic action of various bioactive components such as miRNAs and growth factors. 
Photoaged mouse dorsal skin was caused by ten-week UV irradiation, presenting evident wrinkles and microwrinkles (Figure 5A and $\mathrm{B}$ ). The microwrinkles were significantly reduced after the treatment with hucMSCExos combined with SHSs, and in the case of the high dose of exosomes, the skin appearance even returned to the normal level. Moreover, the combination of hucMSCExos and SHSs alleviated the histopathological changes of photoaged skin, which were represented by epidermal thickening and DEJ flattening (Figure 5C and D). Further study at the molecular level showed that the application of hucMSC-Exos based on SHSs indeed increased the expressions of the ECM genes, collagen I, elastin and fibronectin, and decreased that of the collagenase, MMP1 (Figure 5E). Therefore, hucMSC-Exos in combination with SHSs can produce a desired anti-skin aging effect, while hucMSC-Exos alone hardly do that. These results coincide with our previous observations that exosomes could not easily penetrate through the skin by themselves, but well absorbed in combination with SHSs. Very interestingly, SHSs influenced these gene expressions in a beneficial way as well. The underlying mechanism is supposed to be similar to dermaroller, which has been used to treat scars, skin laxity, wrinkles and pigmentation in clinical practice. Specifically, SHSs could enable skin rejuvenation through microinjuries (with minimal damage to the epidermis) generated by SHSs penetration in the skin. ${ }^{42}$ The microinjuries initiate a wound healing cascade with release of various growth factors, which stimulate the production of extracellular matrix in the papillary layer of the dermis. ${ }^{42}$ Therefore, when combined with hucMSCExos, SHSs not only promote the skin penetration of exosomes but also contribute to stimulating the rejuvenation of the skin.

We also determined the irritation caused by the combination of hucMSC-Exos and SHSs in terms of skin redness and inflammatory cell infiltration. The skin showed very minimal redness one day after the treatment and recovered in the next two days (Figure 6). In the meanwhile, their combined use induced some inflammatory infiltrate immediately, and the latter completely disappeared $24 \mathrm{~h}$ later (Figure 7A and B). Compared to the single use of SHSs, ${ }^{12}$ the combination of hucMSC-Exos and SHSs showed a faster recovery of the skin from the resulting inflammatory infiltration. This might be associated with the immunomodulatory function of MSC-Exos. ${ }^{43}$ Therefore, the combined use of MSC-Exos and SHSs may be considered safe for the skin.

\section{Conclusion}

In this study, MSC-Exos were found not to readily penetrate through the skin, which accords with the theoretical expectation. We validated that SHSs offer a safe and effective way to enhance the skin penetration of MSCExos, and consequently to help them exert the intended therapeutic effects through skin delivery. The combination of MSC-Exos and SHSs can produce significant therapeutic effects against skin photoaging in mice. To our best knowledge, it is the first report on the microneedle-based skin delivery of MSC-Exos or even exosomes. SHSs may also be used to promote the skin delivery of any exosomes. Considering the diversities of exosomes and their biological effects, the skin delivery of exosomes based on SHSs could have great application potential in the medical field.

\section{Acknowledgment}

This study was supported by the National Natural Science Foundation of China (Grant No. 81703939), the China Postdoctoral Science Foundation (Grant Nos. 2017M620403 and 2018T110929), the Fundamental Research Funds for the Central Universities (Grant Nos. 20720180123 and 20720150069), the Science and Technology Planning Project of Fujian Province (Grant No. 2017Y4015), and the Shenzhen Maker Special Fund (Grant No. GRCK20170822152504483).

\section{Disclosure}

Prof. Dr. Ming Chen reports a patent PCT/CN2020/ 073345 pending, a patent US 16/028,036 issued, a patent CN201910958941.9 pending. The authors declare no other conflict of interest.

\section{References}

1. Mahla RS. Stem cells applications in regenerative medicine and disease therapeutics. Int J Cell Biol. 2016;2016:6940283. doi:10.1155/ 2016/6940283

2. Han Y, Li X, Zhang Y, Han Y, Chang F, Ding J. Mesenchymal stem cells for regenerative medicine. Cells. 2019;8(8):886. doi:10.3390/ cells 8080886

3. Gnecchi M, Zhang Z, Ni A, Dzau VJ. Paracrine mechanisms in adult stem cell signaling and therapy. Circ Res. 2008;103(11):1204-1219. doi:10.1161/CIRCRESAHA.108.176826

4. Baraniak PR, McDevitt TC. Stem cell paracrine actions and tissue regeneration. Regen Med. 2010;5(1):121-143. doi:10.2217/rme.09.74

5. Zeringer E, Barta T, Li M, Vlassov AV. Strategies for isolation of exosomes. Cold Spring Harb Protoc. 2015;2015(4):319-323. doi:10.1101/pdb.top074476

6. Thery C, Zitvogel L, Amigorena S. Exosomes: composition, biogenesis and function. Nat Rev Immunol. 2002;2(8):569-579. doi:10.1038/ nri855 
7. Dragomir M, Chen B, Calin GA. Exosomal lncRNAs as new players in cell-to-cell communication. Transl Cancer Res. 2018;7(Suppl S2): S243-S252. doi:10.21037/tcr.2017.10.46

8. Phinney DG, Pittenger MF. Concise review: MSC-derived exosomes for cell-free therapy. Stem Cells. 2017;35(4):851-858. doi:10.1002/ stem. 2575

9. Wiklander OPB, Brennan MA, Lotvall J, Breakefield XO, El Andaloussi S. Advances in therapeutic applications of extracellular vesicles. Sci Transl Med. 2019;11(492):eaav8521. doi:10.1126/scitranslmed.aav8521

10. Mitragotri S. Modeling skin permeability to hydrophilic and hydrophobic solutes based on four permeation pathways. $J$ Control Release. 2003;86(1):69-92. doi:10.1016/S0168-3659(02)00321-8

11. Kim YJ, Yoo SM, Park HH, et al. Exosomes derived from human umbilical cord blood mesenchymal stem cells stimulates rejuvenation of human skin. Biochem Biophys Res Commun. 2017;493(2):11021108. doi:10.1016/j.bbrc.2017.09.056

12. Zhang S, Ou H, Liu C, et al. Skin delivery of hydrophilic biomacromolecules using marine sponge spicules. Mol Pharm. 2017;14 (9):3188-3200. doi:10.1021/acs.molpharmaceut.7b00468

13. Zhang C, Zhang K, Zhang J, et al. Skin delivery of hyaluronic acid by the combined use of sponge spicules and flexible liposomes. Biomater Sci. 2019;7(4):1299-1310. doi:10.1039/C8BM01555D

14. Thery C, Amigorena S, Raposo G, Clayton A. Isolation and characterization of exosomes from cell culture supernatants and biological fluids. Curr Protoc Cell Biol. 2006;30(1):3.22.21-23.22.29. doi:10.1002/0471143030.cb0322s30

15. Karande P, Jain A, Mitragotri S. Relationships between skin's electrical impedance and permeability in the presence of chemical enhancers. J Control Release. 2006;110(2):307-313. doi:10.1016/j. jconrel.2005.10.012

16. Draize JH, Woodard G, Calvery HO. Methods for the study of irritation and toxicity of substances applied topically to the skin and mucous membranes. J Pharmacol Exp Ther. 1944;82(3):377-390.

17. Liu S, Jin MN, Quan YS, et al. Transdermal delivery of relatively high molecular weight drugs using novel self-dissolving microneedle arrays fabricated from hyaluronic acid and their characteristics and safety after application to the skin. Eur J Pharm Biopharm. 2014;86 (2):267-276. doi:10.1016/j.ejpb.2013.10.001

18. Raposo G, Stoorvogel W. Extracellular vesicles: exosomes, microvesicles, and friends. J Cell Biol. 2013;200(4):373-383. doi:10.1083/ jcb.201211138

19. Bazin R, Leveque JL. Longitudinal study of skin aging: from microrelief to wrinkles. Skin Res Technol. 2011;17(2):135-140. doi:10.1111/j.1600-0846.2010.00481.x

20. Limbert G, Kuhl E. On skin microrelief and the emergence of expression micro-wrinkles. Soft Matter. 2018;14(8):1292-1300. doi:10.1039/C7SM01969F

21. Kong SZ, Chen HM, Yu XT, et al. The protective effect of $18 \beta$ Glycyrrhetinic acid against UV irradiation induced photoaging in mice. Exp Gerontol. 2015;61:147-155. doi:10.1016/j. exger.2014.12.008

22. Sanches Silveira JE, Myaki Pedroso DM. UV light and skin aging. Rev Environ Health. 2014;29(3):243-254. doi:10.1515/reveh-20140058

23. Matsumoto T, Ikuta N, Mori M, Nagayama K. Mechanics of wrinkle formation: micromechanical analysis of skin deformation during wrinkle formation in ultraviolet-irradiated mice. Skin Res Technol. 2010;16(2):179-189. doi:10.1111/j.1600-0846.2009.00419.x

24. Tanner T, Marks R. Delivering drugs by the transdermal route: review and comment. Skin Res Technol. 2008;14(3):249-260. doi:10.1111/j.1600-0846.2008.00316.x
25. Kirjavainen M, Urtti A, Jaaskelainen I, et al. Interaction of liposomes with human skin in vitro-the influence of lipid composition and structure. Biochim Biophys Acta. 1996;1304(3):179-189. doi:10.1016/S0005-2760(96)00126-9

26. Prausnitz MR. Engineering microneedle patches for vaccination and drug delivery to skin. Annu Rev Chem Biomol Eng. 2017;8(1):177200. doi:10.1146/annurev-chembioeng-060816-101514

27. Chen W, Li H, Shi D, Liu Z, Yuan W. Microneedles as a delivery system for gene therapy. Front Pharmacol. 2016;7:137. doi:10.3389/ fphar.2016.00137

28. Hao Y, Li W, Zhou X, Yang F, Qian Z. Microneedles-based transdermal drug delivery systems: a review. J Biomed Nanotechnol. 2017;13 (12):1581-1597. doi:10.1166/jbn.2017.2474

29. Bhatnagar S, Dave K, Venuganti VVK. Microneedles in the clinic. $J$ Control Release. 2017;260:164-182. doi:10.1016/j.jconrel.2017. 05.029

30. Ripolin A, Quinn J, Larraneta E, Vicente-Perez EM, Barry J, Donnelly RF. Successful application of large microneedle patches by human volunteers. Int $J$ Pharm. 2017;521(1-2):92-101. doi:10.1016/j.ijpharm.2017.02.011

31. Park JH, Choi SO, Seo S, Choy YB, Prausnitz MR. A microneedle roller for transdermal drug delivery. Eur J Pharm Biopharm. 2010;76 (2):282-289. doi:10.1016/j.ejpb.2010.07.001

32. Lee JW, Prausnitz MR. Drug delivery using microneedle patches: not just for skin. Expert Opin Drug Deliv. 2018;15(6):541-543. doi:10.1080/17425247.2018.1471059

33. Farage MA, Miller KW, Elsner P, Maibach HI. Intrinsic and extrinsic factors in skin ageing: a review. Int J Cosmet Sci. 2008;30(2):87-95. doi:10.1111/j.1468-2494.2007.00415.x

34. Oh M, Lee J, Kim YJ, Rhee WJ, Park JH. Exosomes derived from human induced pluripotent stem cells ameliorate the aging of skin fibroblasts. Int J Mol Sci. 2018;19(6):1715. doi:10.3390/ ijms 19061715

35. Widgerow AD, Fabi SG, Palestine RF, et al. Extracellular matrix modulation: optimizing skin care and rejuvenation procedures. $J$ Drugs Dermatol. 2016;15(4 Suppl):s63-71.

36. Rock K, Fischer JW. Role of the extracellular matrix in extrinsic skin aging. Hautarzt. 2011;62(8):591-597. doi:10.1007/s00105-011-2133$\mathrm{x}$

37. Baek G, Choi H, Kim Y, Lee HC, Choi C. Mesenchymal stem cellderived extracellular vesicles as therapeutics and as a drug delivery platform. Stem Cells Transl Med. 2019;8(9):880-886. doi:10.1002/ sctm.18-0226

38. Yin K, Wang S, Zhao RC. Exosomes from mesenchymal stem/stromal cells: a new therapeutic paradigm. Biomark Res. 2019;7(1):8. doi:10.1186/s40364-019-0159-x

39. Ti D, Hao H, Fu X, Han W. Mesenchymal stem cells-derived exosomal microRNAs contribute to wound inflammation. Sci China Life Sci. 2016;59(12):1305-1312. doi:10.1007/s11427-016-0240-4

40. Liang X, Zhang L, Wang S, Han Q, Zhao RC. Exosomes secreted by mesenchymal stem cells promote endothelial cell angiogenesis by transferring miR-125a. J Cell Sci. 2016;129(11):2182-2189. doi: $10.1242 /$ jcs. 170373

41. Carrasco E, Soto-Heredero G, Mittelbrunn M. The role of extracellular vesicles in cutaneous remodeling and hair follicle dynamics. Int J Mol Sci. 2019;20(11):2758. doi:10.3390/ijms20112758

42. Iriarte C, Awosika O, Rengifo-Pardo M, Ehrlich A. Review of applications of microneedling in dermatology. Clin Cosmet Investig Dermatol. 2017;10:289-298. doi:10.2147/CCID.S142450

43. Borger V, Bremer M, Ferrer-Tur R, et al. Mesenchymal stem/stromal cell-derived extracellular vesicles and their potential as novel immunomodulatory therapeutic agents. Int J Mol Sci. 2017;18(7):1450. doi:10.3390/ijms 18071450 


\section{Publish your work in this journal}

The International Journal of Nanomedicine is an international, peerreviewed journal focusing on the application of nanotechnology in diagnostics, therapeutics, and drug delivery systems throughout the biomedical field. This journal is indexed on PubMed Central, MedLine, CAS, SciSearch ${ }^{\mathbb{B}}$, Current Contents ${ }^{\mathbb{B}} /$ Clinical Medicine, $^{2}$
Journal Citation Reports/Science Edition, EMBase, Scopus and the Elsevier Bibliographic databases. The manuscript management system is completely online and includes a very quick and fair peer-review system, which is all easy to use. Visit http://www.dovepress.com/ testimonials.php to read real quotes from published authors.

Submit your manuscript here: https://www.dovepress.com/international-journal-of-nanomedicine-journal 